\title{
Hand-wringing over handwashing
}

$\mathrm{U}$ ntold numbers of bottles of antibacterial soap and hand cleanser have been purchased, and modern sinks installed. Some facilities have even installed "handprint" screen savers that gently remind staff to start scrubbing, while others have gone so far as to appoint "secret observers" to lurk in the shadows and report to administrators whether health workers are following hand hygiene protocols.

Yet the Canadian Patient Safety Institute (CPSI) says health worker compliance with optimal hand-hygiene practices continues to hover under $40 \%$ while health care-associated infections affect some 220000 Canadians, killing more than 8000 annually (www.hand hygiene.ca/English/Documents/Fact\%20 Sheets/Fact $\% 20$ Sheet $\% 201 \% 20$ The $\% 20 \mathrm{Need} \% 20$ for $\% 20 \mathrm{Better} \% 20 \mathrm{Hand}$ $\% 20$ Hygiene.pdf).

The latest solution? Physician, nurse, etc., clean thyself.

It seems some infection control experts are embracing a sink-or-swim notion - that the solution to all those little bugs crawling over the fingers of physicians and nurses lies in the hands of frontline staff. To wit, they should craft their own strategies for improving hand hygiene, as long as they are prepared to have the results reported publicly.

Allowing staff to take ownership of the problem may be far more effective than measures dictated by infection control divisions, says Dr. Michael Gardam, physician director of the Community and Hospital Infection Control Association Canada and Director of Infection Prevention and Control at University Health Network (UHN) in Toronto, Ontario. "It's a very complex problem that requires multiple strategies and they need to be locally owned strategies rather than one-size-fits-all."

External interventions such as posters and pamphlets have had disappointing results, says Anne MacLaurin, project manager of CPSI's flagship pro-

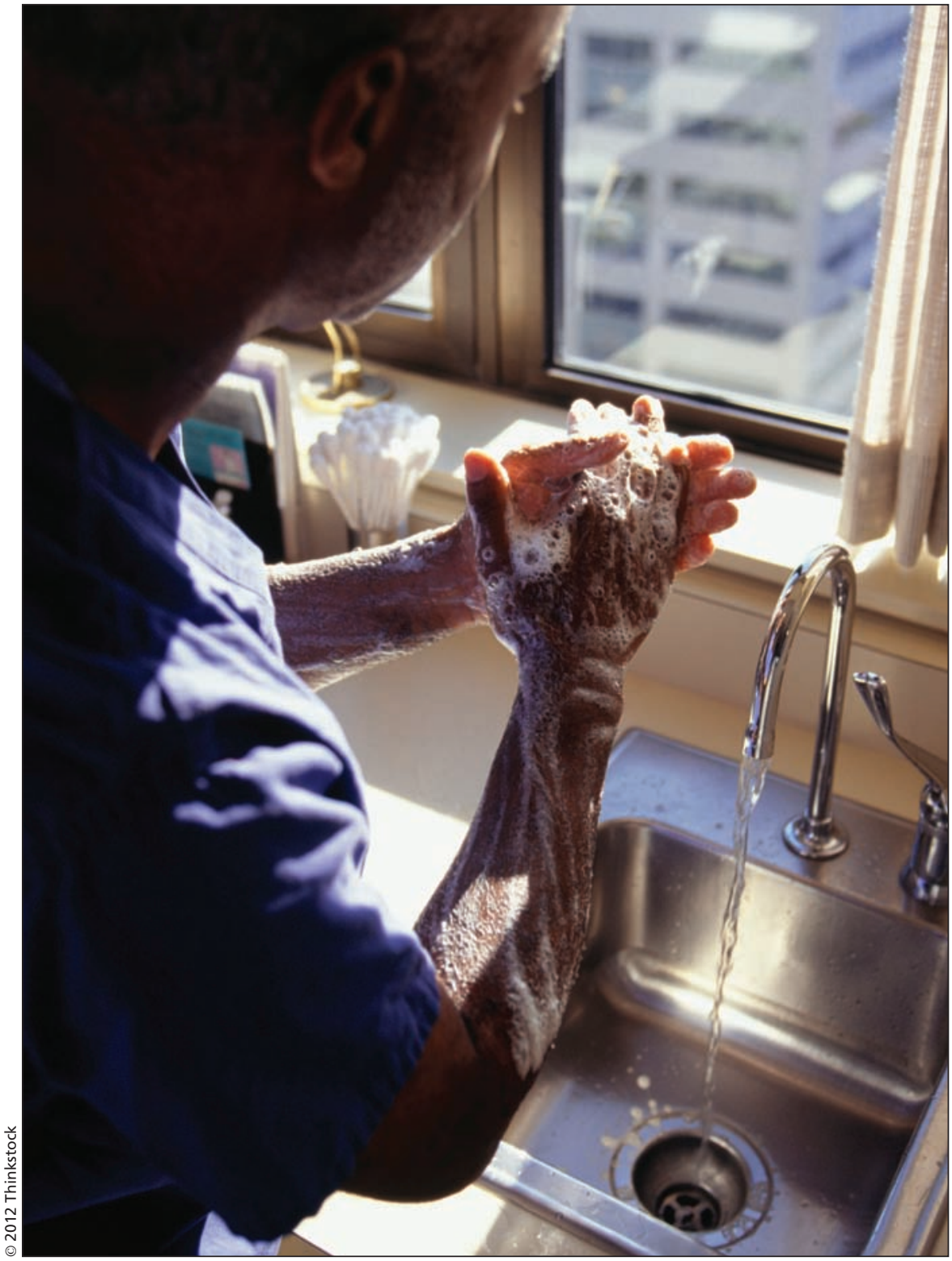

Despite posters, pamphlets and other reminders, health worker compliance with optimal hand-hygiene practices continues to hover under $40 \%$.

gram to reduce avoidable harm, Safer Healthcare Now! "Take that away and it becomes everybody's responsibility."

Accountability mechanisms are more effective when driven by staff, concurs Tobie Guinez, a clinical nurse educator at the Mazankowski Alberta Heart Institute in Edmonton. "I think whenever you go out there and you're making changes and you're deciding what's going to work for front-line staff who are out there in the rooms every day, it doesn't work. The ideas need to come from them. They need to think about what's important and how it's going to work for them.'

The role of infection-control managers within such a model is to primarily focus on "setting clear targets, doing good auditing and feeding back infor- 
mation very publicly," Gardam says. "The work we've been doing at UHN the last couple of years is really about telling people where they need to get to, but not telling individual units how to get there."

It's a marked departure from the educational approaches of decades past, Gardam adds. Those missed the "big elephant in the room" - that the current culture tolerates spotty compliance. "Hygiene in general is still not at a point where a lot of organizations get it. They see it as a check box: 'We've focused on hand hygiene for six months. We've had our campaign. Check."”

About the only area where hygiene is truly the rule rather than the exception is the operating room (OR), says Elaine Larson, associate dean for research and professor of therapeutic and pharmaceutical research at the Columbia University School of Nursing in New York City, New York. "If you go into the OR and you don't do a pre-op skin prep, people will kick you out. It doesn't matter if you're the chief surgeon. It's the culture. Yet, it's not the culture in our regular units or even in our intensive care units. A nurse would never or very rarely say to a doctor, 'You didn't wash your hands.'”

Many health care workers lack a sense of their personal responsibility in infection prevention, Larson says. "The main barrier is that hand hygiene has to be done so many times every day and there's very little immediate feedback when it's successful. In other words, if a surgeon cuts the wrong artery, the patient bleeds and there's immediate feedback. If a patient gets an infection three days from now, you don't have direct evidence that it was you not doing correct hand hygiene today."

Gardam says most front-line staff perceive hand-hygiene campaigns as targeted at others and merely redundant. "Our previous model of infection control was largely me nagging you to the point where basically you tune me out. Nagging doesn't work, surprisingly."

When the UHN asked staff to take ownership of the issue, "some of them spin their wheels for months not getting that they're expected to improve, but others get it right away and jump on it," he adds. But when the results began being posted, the laggards caught on quickly. "If you walk onto one of our nursing units, there's a sign that posts the hand-hygiene rate for the last month. We don't hide it from anybody and send it around so programs know where they are ranked compared to others."

Hand-hygiene compliance has shot up from about $30 \%$ to more than $80 \%$ in a year. It's a "huge increase," Gardam says. "I've seen a real fundamental organizational culture shift."

That approach is now gaining traction at other facilities, MacLaurin says. "It's not so much about what [specific interventions] they decide to test out. It's more or less how they come up with the different ideas to test, so they take on a different type of ownership because it's their own idea."

Other successful interventions have included front-line staff at Alberta Health Services' hospitals using stickers to identify where hand sanitizer dispensers were needed, and wearing buttons to encourage patients and colleagues to ask them, point blank, if their hands were clean, Guinez says. "Patients started asking questions like, 'How come you have a button and somebody else doesn't?' It also made patients feel more comfortable to say to their health care providers, 'Can you wash your hands before you come in?"”
At UHN, one nurse manager bought breakfast for her team when they improved their hand hygiene, while another undertook rounds with lackadaisical handwashers, offering to clean surgeons' hands for them. "It was a very sarcastic approach which worked really well with those surgeons. They started to joke about it, clean their hands more often and then do it without her bugging them," Gardam says. "It had all these downstream effects to the point that the unit is now one of the best for hand hygiene in the organization, and they certainly didn't start out that way."

"But if I sent around an email to all of our nurse managers saying, 'Please sarcastically wash your doctors' hands,' that wouldn't go over. It worked because that's the relationship she had and that's what she came up with," he adds.

At the Toronto East General Hospital, even housekeeping staff got involved, tagging high-touch surfaces with a "72-hour best-before date" after cleaning (www.stopsuperbugs.com /contents/december-edition/pd-at-tegh -producing-results-superbug). Once the tag expires, the tag can be removed by anyone to remind the cleaners that the item again needs attention. As a result of such efforts, the hospital documented a $64 \%-70 \%$ reduction in Clostridium difficile infections.

Waiting for decentralized, local solutions can be a challenge for impatient infection control managers, Gardam says. But it's a misconception that the solution "is going to be easy and you can do it in six months. It's actually going to take years to really fundamentally improve practice to the point where you can actually see a difference when you walk onto a unit." - Lauren Vogel, CMAJ

CMAJ 2012. DOI:10.1503/cmaj.109-4222 